Short communication

\title{
Dynamic feet distance: A new functional assessment during treadmill locomotion in normal and thoracic spinal cord injured rats
}

\author{
Camila Cardoso Diogo ${ }^{\mathrm{a}}$, Luís Maltez da Costa ${ }^{\mathrm{a}, \mathrm{b}}$, José Eduardo Pereira ${ }^{\mathrm{a}, \mathrm{b}}$, Vítor Filipe ${ }^{\mathrm{c}, \mathrm{d}}$, \\ Pedro Alexandre Couto ${ }^{\mathrm{c}, \mathrm{e}}$, Luís G. Magalhães ${ }^{\mathrm{f}}$, Stefano Geuna ${ }^{\mathrm{g}}$, Paulo A. Armada-da-Silva ${ }^{\mathrm{h}, \mathrm{i}}$, \\ Ana Colette Maurício ${ }^{\mathrm{j}, \mathrm{k}}$, Artur Severo Varejão ${ }^{\mathrm{a}, \mathrm{b}, *}$ \\ a Department of Veterinary Sciences, University of Trás-os-Montes e Alto Douro, UTAD, Quinta de Prados, 5000-801 Vila Real, Portugal \\ b CECAV, Centre for Animal Sciences and Veterinary Studies, University of Trás-os-Montes e Alto Douro, Quinta de Prados, 5000-801 Vila Real, Portugal \\ ${ }^{\mathrm{c}}$ Department of Engineering, School of Science and Technology, University of Trás-os-Montes e Alto Douro, UTAD, Quinta de Prados, 5000-801 Vila Real, Portugal \\ d INESC TEC, Rua Dr. Roberto Frias, 4200 - 465 Porto, Portugal \\ e CITAB, Centre for the Research and Technology of Agro-Environmental and Biological Sciences, University of Trás-os-Montes e Alto Douro, Quinta de Prados, 5000-801 \\ Vila Real, Portugal \\ ${ }^{f}$ Centro Algoritmi, University of Minho, Campus Azurém, 4800-058 Guimarães, Portugal \\ ${ }^{g}$ Department of Clinical and Biological Sciences, University of Turin, Italy \\ ${ }^{\text {h }}$ Faculdade de Motricidade Humana (FMH), Universidade de Lisboa (ULisboa), Estrada da Costa, 1499-002, Dafundo, Cruz Quebrada, Portugal

 \\ Costa, 1499-002, Cruz Quebrada - Dafundo, Portugal \\ ${ }^{\mathbf{j}}$ Department of Veterinary Clinics, Institute of Biomedical Sciences Abel Salazar (ICBAS), University of Porto (UP), Rua de Jorge Viterbo Ferreira, $n^{\circ} 228$, 4050-313 \\ Porto, Portugal \\ ${ }^{\mathbf{k}}$ Animal Science and Study Centre (CECA), Institute of Sciences, Technologies and Agroenvironment of the University of Porto (ICETA), Rua D. Manuel II, Apartado \\ 55142, 4051-401, Porto, Portugal
}

\section{A R T I C L E I N F O}

\section{Keywords:}

Rat

Spinal cord injury

3D gait analysis

Kinematics

Locomotion

Dynamic feet distance

\begin{abstract}
A B S T R A C T
Of all the detrimental effects of spinal cord injury (SCI), one of the most devastating is the disruption of the ability to perform functional movement. Very little is known on the recovery of hindlimb joint kinematics after clinically-relevant contusive thoracic lesion in experimental animal models. A new functional assessment instrument, the dynamic feet distance (DFD) was used to describe the distance between the two feet throughout the gait cycle in normal and affected rodents. The purpose of this investigation was the evaluation and characterization of the DFD during treadmill locomotion in normal and T9 contusion injured rats, using threedimensional (3D) instrumented gait analysis. Despite that normal and injured rats showed a similar pattern in the fifth metatarsal head joints distance excursion, we found a significantly wider distance between the feet during the entire gait cycle following spinal injury. This is the first study to quantify the distance between the two feet, throughout the gait cycle, and the biomechanical adjustments made between limbs in laboratory rodents after nervous system injury.
\end{abstract}

Although the incidence of acute spinal cord injury (SCI) is low, represents one of the leading causes of disabilities in young adult with limited recovery of locomotor activity. As SCI related muscular and neural impairments affect the legs, gait analysis is often used as a measure of impairment and recovery. Affected persons report balance dysfunction, associated to wide base-of-support, as a contributor to the high falls incidence and second injuries [1]. Rats are commonly used for the study of movement disorders in spinal cord injury research [2]. In the last two decades several researchers have collected spatial and temporal step kinematics in spinal cord injured rats. Spinal cord lesions in rats have been described to increase the base of support and decrease the stride length [3]. The base of support is a basic gait parameter that has been traditionally studied through footprint analysis [3], but recent advances in automated gait analysis have enabled researchers to quickly and easily obtain this spatial parameter of gait [4]. Nevertheless, recording step width has been restricted to measure the distance perpendicular to the trajectory between the two hindlimbs during the stance phase of walking. Surprisingly, there is no data describing the distance between the two feet throughout the gait cycle in normal or affected rodents. Given the dynamic nature of walking, an expansion

\footnotetext{
* Corresponding Author: Department of Veterinary Sciences, University of Trás-os-Montes e Alto Douro, UTAD, Quinta de Prados, 5000-801 Vila Real, Portugal.

E-mail address: avarejao@utad.pt (A.S. Varejão).
} 
of this static spatial measurement seems critical in order to fully characterize rat locomotion. Thus, the current study was designed to examine and characterize the dynamic feet distance (DFD) during treadmill locomotion in normal and thoracic spinal cord injured rats. Since persons with SCI may require corrective foot placements to maintain stability in the face of sensorimotor impairments, DFD findings could provide a window into biomechanically how these individuals are able to accomplish the task.

Ten Wistar adult female rats (Harlan, Barcelona, Spain) weighing approximately $200 \mathrm{~g}$ were used in this study. Data were collected from a total of eight animals due to the training protocol (see training procedure). All animals were kept in ventilated, humidity and temperaturecontrolled rooms with a 12/12-h light/dark cycle. The animals were housed on sawdust and received food pellets and water ad lib. All procedures were performed with the approval of the Portuguese Veterinary Authorities, in accordance with the EU Directive 2010/63/ EU for animal experiments.

Rats were anesthetized with ketamine $(80 \mathrm{mg} / \mathrm{kg})$ and medetomidine $(0.2 \mathrm{mg} / \mathrm{kg})$. A midline incision exposed the spinal column at the T8-T11 level, and the paravertebral muscles were dissected bilaterally to visualize the transverse apophyses. Care was taken to perform a laminectomy at the T10 vertebral level that was only slightly larger than the $2.5 \mathrm{~mm}$ impactor tip. The animals then received a 200-kdyne contusion with the Infinite Horizon spinal cord injury device (Precision Systems \& Instrumentation, Lexington, KY, USA). The surgical procedure was performed with the aid of an operating microscope (M680, Leica, Wetzlar, Germany). The wound was subsequently irrigated with saline solution, the muscle, the fascia and the skin were reapproximated with absorbable sutures. Body temperature was maintained with a heating pad at $37 \pm 0.5^{\circ} \mathrm{C}$ during the surgery and postoperative period. An ophthalmic gel (Lacryvisc, Alcon, Lisbon, Portugal) was applied to prevent drying of the eyes.

After surgery, $10 \mathrm{ml}$ of $0.9 \%$ sodium chloride and $30 \mathrm{mg} / \mathrm{kg}$ of sulfadiazine and trimethoprim were injected subcutaneously. The animal's access to food was facilitated by placing softened food pellets directly in the bottom of each cage. The state of hydration and gastrointestinal function were monitored daily. Lactated Ringer's solution was injected subcutaneously when necessary. Rats were weighed daily for the first 7 days postsurgery and then weighed weekly. Postoperative care included the manual expression of bladders twice a day until bladder function returned, as well as injections of sulfadiazine and trimethoprim twice a day for up to 1 week.

Two weeks before the collection of data rats were trained daily to walk consistently on a treadmill at a speed of $40 \mathrm{~cm} / \mathrm{s}$, within a Plexiglas enclosure, $53 \mathrm{~cm} \times 10 \mathrm{~cm} \times 14 \mathrm{~cm}$, with a removable top (Letica, Scientific Instrument, Barcelona, Spain). Ten minute long training sessions were given once a day with mild intensities of footshock initially used as negative reinforcement to improve performance. In our training protocol when the rats failed to walk regularly and spontaneously after three sessions, they were eliminated from further study. We attempted to minimize the stress by minimizing noise levels and handling the rats gently to obtain locomotion under normal conditions.

Kinematic data were collected before spinal injury (week 0) and 3 weeks postoperatively. Hemispherical green markers with a diameter of $2 \mathrm{~mm}$ were placed on the skin over the fifth metatarsal head of the left and right hindlimbs. The same operator performed all marker placements to avoid inter-tester variability. The coloured passive markers were used together with appropriate illumination produced by four fluorescent high frequency lamps (StockerYale 13W, $25 \mathrm{Khz}$, Prophotonix, Inc, Salem, USA) mounted around the capture volume.

Three-dimensional (3D) video recordings were made with four CMOS cameras (PhotonFocus MV-D640C, Lachen, Switzerland) oriented at $45^{\circ}$ with respect to the direction of the locomotion (that is, the rat's sagittal plane) on both sides. Kinematic data were collected at a sampling rate of $144 \mathrm{~Hz}$. The cameras' field of view was calibrated to cover a $20 \mathrm{~cm}$ length of the treadmill apparatus, and allowed the recording of a complete gait cycle. Data were recorded while rats walked at $40 \mathrm{~cm} / \mathrm{s}$, which are within the normal walking velocity, where typically they utilize a lateral sequence walk [5]. The images were acquired using the software Video Savant 4 (IO Industries Inc, Ontario, Canada). The colour image had a resolution of $640 \times 480$ pixels. Cameras were strategically placed around the animal to minimize marker occlusion, maximize resolution and to improve the accuracy of the $3 \mathrm{D}$ reconstruction process. We designed a new calibration object, which allowed us to calibrate all the four cameras at the same time. The use of the calibration object ensured that the projection matrixes were all related to the same coordinate system. This allowed all points to be used to compute the kinematic parameters without any need for their transformation. The 3D coordinates obtained were compared with 3D coordinates measured in the calibration object and for each point a position error was calculated. This error was computed as the Euclidean distance between the measured and the reconstructed 3D coordinate. We obtained a mean absolute position error of $0.41 \pm 0.3 \mathrm{~mm}$. The camera calibration and the 3D reconstruction process for each side followed a procedure similar to the one previously described in detail [6].

DFD was quantified by measuring the perpendicular distance between the left and right foot by tracking the 3D locations of markers secured over the fifth metatarsal head joints throughout the gait cycle, which was defined by the initial-contact (IC) of the left foot (Fig. 1).

The gait cycle, which is the basic unit of measurement in gait analysis, was split into two parts, the stance and the swing phases. The stance phase was defined as the part of the gait cycle that begins as soon as the foot contacts the treadmill belt, and ceases when the foot is lifted from the treadmill belt and starts its forward movement. The swing phase was considered to begin at the onset of forward movement and to terminate as the foot strikes the treadmill belt. For each stride, the duration of the stance and swing phases was normalized. Cubic-spline interpolation was applied to the original data about the position of fifth metatarsal head to obtain 101 samples per gait cycle regardless of their duration [7]. This numerical treatment was performed with Matlab computational software (The MathWorks Inc., Natick, MA, USA).

Mean \pm standard deviation (S.D.) values for all of the measured variables are reported. Differences in DFD values before and after spinal cord contusion were tested for significance with paired Student's $t$-test and with the Wilcoxon signed-rank test when data were not normally distributed. The statistical significance was set at the level of $P<0.05$. These statistical tests were performed using SPSS v. 22 computational software (Statistical Package for the Social Sciences Inc., Chicago, USA).

All of the contused animals exhibited signs of paraplegia, followed

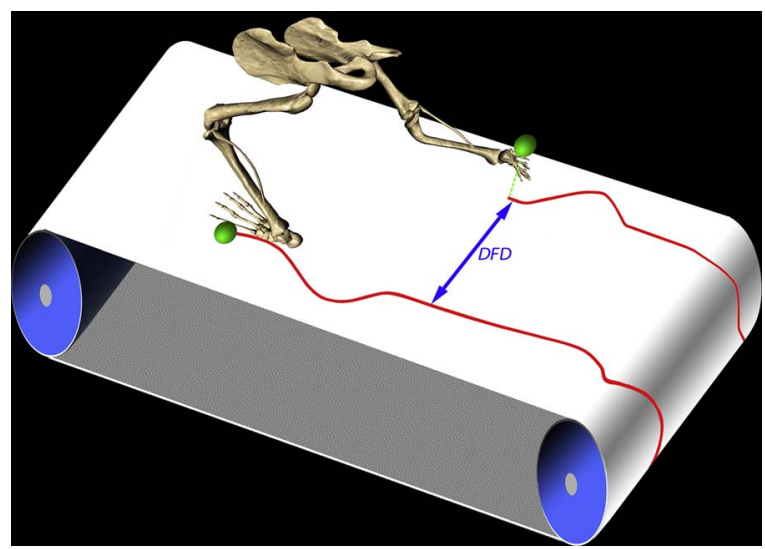

Fig. 1. The dynamic feet distance (DFD) is quantified by measuring the perpendicular distance between the left and right foot by tracking the 3D locations of markers secured over the fifth metatarsal head joints throughout the gait cycle. 


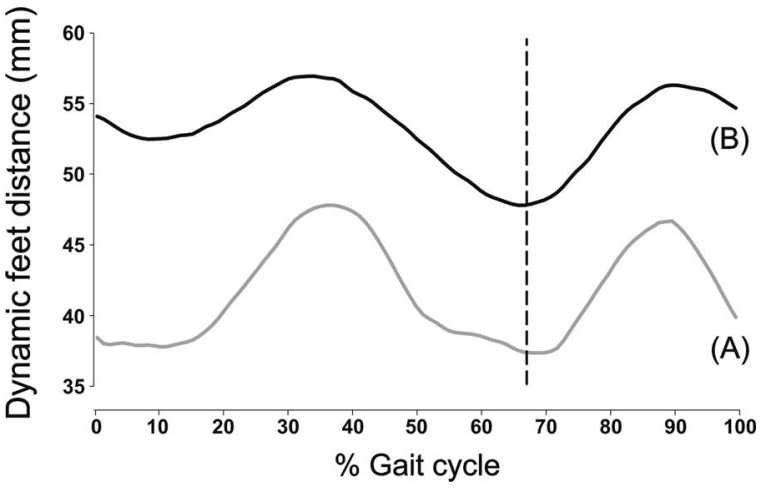

Fig. 2. Computerized analysis of 3D coordinates of the fifth metatarsal head joints markers were used to calculate the mean values for the dynamic feet distance (DFD), before (grey trace) (A) and 3 weeks following SCI (black trace) (B) during treadmill locomotion. Stance and swing phases were normalized. The stance duration was set at $67 \%$ of the gait cycle duration. The vertical dashed line corresponds to the stance-swing transition.

by significant improvement over the subsequent 3 weeks. At week-3 postoperatively they could perform weight supported plantar steps in the treadmill at a speed of $40 \mathrm{~cm} / \mathrm{s}$.

To characterize the 3D DFD during treadmill locomotion, a total of 80 gait cycles (10 for each animal) were obtained before and 3 weeks after spinal cord contusion injury. The average toe-off time was calculated to be $67 \%$ into the gait cycle. The kinematic curves of DFD are shown in Fig. 2. During normal control gait, DFD varied during the gait cycle, peaking at approximately the midstance and midswing time points. Again in the control gait, the minimal DFD values occurred at the late stance and early swing and, therefore, in coincidence with the stance-to-swing transition (see Fig. 2). No significant differences for maximum (MAX) and minimum (MIN) DFD values between the stance and swing phases or between IC and toe-off (TO) existed during normal gait $(P>0.05)$. Three weeks after SCI, rats showed a significant increase in DFD during gait. After spinal cord contusion, a significant increase in MAX and MIN DFD values during both swing and stance and in DFD values at IC and TO could be registered (all comparisons $P<0.01$ ), demonstrating that the distance between the feet was wider during the entire gait cycle following injury. Additionally, increased DFD value at IC compared to the value at TO could also be found after spinal cord contusion $(P<0.05)$ (Fig. 2).

The present study provided the first accurate measure of the continuous distance between the left and right foot of healthy and thoracic spinal injured rats walking on a treadmill. The spatial accuracy of the DFD was obtained by measuring the actual 3D movements of the fifth metatarsal head joints, and the temporal accuracy was provided by the high speed digital cameras, acquiring a new image every $7 \mathrm{~ms}$.

The animal model of contusion injury that we employed mimics the typical injury mechanism in humans [8]. In our study the injury was delivered with a force of 200-kdyne using the Infinite Horizon spinal cord injury device. This moderate injury was chosen in order to allow, at week-3 postoperatively, repetitive plantar stepping in the treadmill [9].

Adequate evaluation of the efficacy of therapeutic interventions following spinal injury is critical in order to distinguish among spontaneous recovery, compensatory strategies, and recovery of locomotor function dependent on the therapeutic measure [10]. At this time, the 21-point Basso, Beattie and Bresnahan (BBB) locomotor recovery scale [11] is the most widely used visual gait assessment after thoracic lesion. Despite the fact that BBB locomotor recovery scale covers a broad range of functional recovery, it can be less sensitive at specific levels of recovery, in part due to the ordinal nature of the scale and to the difficulty in correctly assessing the forelimb-hindlimb coordination [12]. It is now well accepted that kinematic gait analysis allows for a more accurate assessment of gait deviations than visual assessment and is widely accepted as a useful research tool $[2,13]$.

This study is the first, to the authors' knowledge, to detect significant changes, throughout the gait cycle, in the distance between the left and right foot of normal and spinal injured rats during treadmill walking. A prominent finding of the present work is that rats walked on a treadmill with significantly wider distance between the feet during the entire gait cycle following spinal injury. From an experimental standpoint, DFD parameter is easy to measure, and the treadmill protocol is easy to administer. While we have confidence in our results for treadmill walking, the influence of walking overground with respect to DFD is unclear. Previously, our research group did a comparison analysis of hindlimb kinematics during treadmill and overground locomotion in rats and found that reliable kinematic measures can be obtained from the treadmill gait analysis [14]. Spinal cord injury results in gait deficits that are commonly assessed with changes in kinematic measures. However, many of these kinematic variables are velocity dependent, making it difficult to compare accurately fast moving healthy rodents to slower walking injured animals. One of the main advantages of treadmill is that walking velocity can be more accurately standardized and therefore matched between experimental groups. The speed of the treadmill was set according to the preferred overground velocity $(\sim 40 \mathrm{~cm} / \mathrm{s})$. Moreover, the speed of $40 \mathrm{~cm} / \mathrm{s}$ is within the range of normal walking velocity of the rat, where they typically utilize a lateral sequence walk [5].

In rat gait analysis, soft tissue movement around the joints has been considered the principle source of error when estimating hindlimb joint kinematics [15]. In our study the hemispheric markers were positioned over palpable bony landmarks, the fifth metatarsal heads. Therefore, this biomechanical model has the advantage of greatly minimizing the error due to marker placement caused by uncertainty in the anatomical landmark position determination and is not prone to soft-tissue artefact as it is not enveloped by bulky muscles.

The two-dimensional (2D) kinematic approach, requiring only one camera to record the movement, is only accurate when the hindlimb joints lie in a single plane that is parallel to the imaging plane. Therefore, these kinematic trajectories can be misleading and one must be careful when interpreting planar representations that have their origin in 3D motion [16]. Our four-camera motion capture system allowed us to perform a 3D reconstruction of the synchronized movement of both feet of normal and spinal injured rats during treadmill walking.

It is remarkable that the SCI rats showed a similar pattern in the fifth metatarsal head joints distance excursion to normal rats. However, during treadmill walking, we clearly demonstrated that injured rats preferred a wider step width along the gait cycle compared to healthy rats. One limitation in this study is the lack of a control group, although having the animals well familiarized with treadmill walking before starting data collection has minimized the possible confounding effect of learning. Neurological affected humans with reduced sensory information from the lower limbs and poor muscle strength to control leg movements also show wider step width [17]. Many studies have suggested that medial-lateral stability is very important for people to maintain balance and avoid falling [18]. The step width variability represents the more sensitive descriptor of locomotion control of older and young adults. By increasing the width of a step, the base of support becomes wider, reducing fall risk [19,20]. It should be noted that when this spatiotemporal parameter increases the lower extremity biomechanics at the hip and knee joint are affected and this will result in additional energy expenditure $[21,22]$. In this study we looked only to feet distance during the treadmill gait. However, in the future other features may be studied, such as the amount of variation of DFD during the gait cycle and its dynamics. This more thourough analysis may provide easy and valuable assessment about recovery of gait following spinal cord injury in the rodent. For example, while a wider stepping may be envisaged as a compensatory strategy to improve balance, altered pattern of base support changes during the step might else indicate diminished muscle power ability and loss of hindlimb trajectory 
control after spinal cord injury.

In summary, this study is the first providing novel information about the distance between the two feet, throughout the gait cycle, before and after a nervous system injury in rats. We have shown that injured rats walked on a treadmill with significantly larger DFD, perhaps to increase their lateral base of support, thereby providing a larger margin of safety for side-to-side motion of the centre of mass. We encourage the application of this biomechanical model to different behavioural contexts and to experimental central and peripheral nervous system lesions or treatment conditions for which accurate locomotor measures are required.

\section{Acknowledgements}

This research was supported by the Project UID/CVT/00772/2013 from FCT.

\section{References}

[1] S.S. Brotherton, J.S. Krause, P.J. Nietert, Falls in individuals with incomplete spinal cord injury, Spinal Cord 45 (2007) 37-40.

[2] O. Alluin, S. Karimi-Abdolrezaee, H. Delivet-Mongrain, H. Leblond, M.G. Fehlings, S. Rossignol, Kinematic study of locomotor recovery after spinal cord clip compression injury in rats, J. Neurotrauma 28 (2011) 1963-1981.

[3] G.A. Metz, D. Merkler, V. Dietz, M.E. Schwab, K. Fouad, Efficient testing of motor function in spinal cord injured rats, Brain Res. 883 (2000) 165-177.

[4] W.T. Hendriks, R. Eggers, M.J. Ruitenberg, B. Blits, F.P. Hamers, J. Verhaagen, G.J. Boe, Profound differences in spontaneous long-term functional recovery after defined spinal tract lesions in the rat, J. Neurotrauma 23 (2006) 18-35.

[5] G.B. Gillis, A.A. Biewener, Hindlimb muscle function in relation to speed and gait: in vivo patterns of strain and activation in a hip and knee extensor of the rat (Rattus norvegicus), J. Exp. Biol. 204 (2001) 2717-2731.

[6] P.A. Couto, V.M. Filipe, L.G. Magalhães, J.E. Pereira, L.M. Costa, P. Melo-Pinto, J. Bulas-Cruz, A.C. Maurício, S. Geuna, A.S.P. Varejão, A comparison of two-dimensional and three-dimensional techniques for the determination of hindlimb kinematics during treadmill locomotion in rats following spinal cord injury, J. Neurosci. Methods 173 (2008) 193-200.

[7] A.S.P. Varejão, A.M. Cabrita, M.F. Meek, J. Bulas-Cruz, R.C. Gabriel, V.M. Filipe,
P. Melo-Pinto, D.A. Winter, Motion of the foot and ankle during the stance phase in rats, Muscle Nerve 26 (2002) 630-635.

[8] M.D. Norenberg, J. Smith, A. Marcillo, The pathology of human spinal cord injury: defining the problems, J. Neurotrauma 21 (2004) 429-440.

[9] J.A. Nessler, K. Minakata, K. Sharp, D.J. Reinkensmeyer, Robot-assisted hindlimb extension increases the probability of swing initiation during treadmill walking by spinal cord contused rats, J. Neurosci. Methods 159 (2007) 66-77.

[10] G.D. Muir, A.A. Webb, Mini-review: assessment of behavioural recovery following spinal cord injury in rats, Eur. J. Neurosci. 12 (2000) 3079-3086.

[11] D.M. Basso, M.S. Beattie, J.C. Bresnahan, A sensitive and reliable locomotor rating scale for open field testing in rats, J. Neurotrauma 12 (1995) 1-21.

[12] G.C. Koopmans, R. Deumens, W.M. Honig, F.P. Hamers, H.W. Steinbusch, E.A. Joosten, The assessment of locomotor function in spinal cord injured rats: the importance of objective analysis of coordination, J. Neurotrauma 22 (2005) 214-225.

[13] L.M. Costa, J.E. Pereira, V.M. Filipe, P.A. Couto, L.G. Magalhães, J. Bulas-Cruz, A.C. Maurício, S. Geuna, A.S.P. Varejão, The effect of gait speed on three-dimensional analysis of hindlimb kinematics during treadmill locomotion in rats, Rev. Neurosci. 21 (2010) 487-497.

[14] J.E. Pereira, A.M. Cabrita, V.M. Filipe, J. Bulas-Cruz, P.A. Couto, P. Melo-Pinto, L.M. Costa, S. Geuna, A.C. Maurício, A.S.P. Varejão, A comparison analysis of hinlimb kinematics during overground and treadmill locomotion in rats, Behav. Brain Res. 172 (2006) 212-218.

[15] V.M. Filipe, J.E. Pereira, L.M. Costa, A.C. Maurício, P.A. Couto, P. Melo-Pinto, A.S. Varejão, Effect of skin movement on the analysis of hindlimb kinematics during treadmill locomotion in rats, J. Neurosci. Methods 153 (2006) 55-61.

[16] A.K. Thota, S.C. Watson, E. Knapp, B. Thompson, R. Jung, Neuromechanical control of locomotion in the rat, J. Neurotrauma 22 (2005) 442-465.

[17] C.L. Chang, B.D. Ulrich, Lateral stabilization improves walking in people with myelomeningocele, J.Biomech. 41 (2008) 1317-1323.

[18] A. Schinkel-Ivy, J.S. Wong, A. Mansfield, Balance confidence is related to features of balance and gait in individuals with chronic stroke, J. Stroke Cerebrovasc. Dis. 26 (2017) 237-245

[19] X. Lin, O.G. Meijer, J. Lin, W. Wu, X. Lin, B. Liang, J.H. van Dieën, S.M. Bruijn, Frontal plane kinematics in walking with moderate hip osteoarthritis: stability and fall risk, Clin. Biomech. (Bristol Avon) 30 (2015) 874-880.

[20] T.M. Owings, M.D. Grabiner, Step width variability but not step length variability or step time variability, discriminates gait of healthy young and older adults during treadmill locomotion, J. Biomech. 37 (2004) 935-938.

[21] R.A. Brindle, C.E. Milner, S. Zhang, E.C. Fitzhugh, Changing step width alters lower extremity biomechanics during running, Gait Posture 39 (2014) 124-128.

[22] S.E. Jansen, A. Toet, P.J. Werkhoven, Human locomotion through a multiple obstacle environment: strategy changes as a result of visual field limitation, Exp. Brain Res. 212 (2011) 449-456. 